\title{
MACROSCOPIC QUANTUM EFFECTS IN BIOPHYSICS AND CONSCIOUSNESS
}

\author{
Dejan Raković ${ }^{1,4}$, Miroljub Dugic ${ }^{2,4}$, Milan M. Ćirković ${ }^{3}$ \\ ${ }^{1}$ Faculty of Electrical Engineering, P.O.Box 35-54, Belgrade, Serbia, Yugoslavia \\ ${ }^{2}$ Faculty of Science, Department of Physics, P.O.Box 60, Kragujevac, Serbia, Yugoslavia \\ ${ }^{3}$ Astronomical Observatory, Volgina 7, Belgrade, Serbia, Yugoslavia \\ ${ }^{4}$ International Anti-Stress Center (IASC), Smiljaniceva 11, Belgrade, Serbia, Yugoslavia \\ info@iasc-bg.org.yu www.iasc-bg.org.yu
}

\begin{abstract}
It is shown that investigations in the field of microwave resonance stimulation of the acupuncture system, as well as investigations of the interactions of consciousness with microscopic and macroscopic environment - imply the existence of local and nonlocal macroscopic quantum biophysical effects, with great potential implications in medicine, psychology, biology, physics, engineering, and philosophy/religion. The parallels between classical and quantum Hopfield-like neural networks were also emphasized, as well as biophysical quantum-relativistic model of altered and transitional states of consciousness, the roots of relative metatheory of consciousness in the quantum decoherence theory, and anthropic "coincidences" in classical and quantum cosmology.
\end{abstract}

Keywords: biophysics; macroquantum local and nonlocal effects; holistic medicine and psychotherapy; acupuncture system \& microwave resonance therapy (MRT); classical \& quantum Hopfield-like neural networks; nonlinearity and nonlocality of the wave function collapse \& macroquantum aspects of consciousness; altered and transitional states of consciousness \& quantum-relativistic model; quantum decoherence theory \& relative metatheory of consciousness; classical and quantum cosmology \& anthropic "coincidences". 


\section{INTRODUCTION}

One century elapsed from the first discoveries of the quantum aspects of Nature, and quantum laws of microworld have got enormous technological applications in semiconductor microelectronics and measurements, optoelectronics and laser technique, computers and telecommunications, with a tendency of further expansion through miniaturization of semiconductor devices till nanometer dimensions. Discoveries of low-temperature and high-temperature semiconductors revealed that quantum effects can be macroscopic as well, with possibility of much wider application of quantum technologies, especially in electroenergetics and transport, and also in microelectronics and measurements of ultrahigh speed and accuracy.

Initially, quantum mechanics appeared as a theory of microscopic physical systems (elementary particles, atoms, molecules) and phenomena at small space-time scales; typically, quantum phenomena are manifested at dimensions smaller than $1 \mathrm{~nm}$ and time intervals shorter than $1 \mu \mathrm{s}$. However, from the very beginning of the quantum-mechanical founding the question of its universality was raised, i.e. the question of general validity of the quantum-physical laws for macroscopic phenomena as well, usually treated by the methods of classical physics. In the history of quantum physics, and especially quantum mechanics, this question has been temporarily put aside for very different reasons, being considered as a difficult scientific problem. The situation is additionally complicated by the existence of different schools of quantum mechanics, arguing about physical-epistemological status of the so called collapse (reduction) of the wave function. In this respect the situation is not much better today, and it can be said freely that the problem of universal validity of quantum mechanics is still open.

Starting from the 1980's, mainly in the papers of Leggett [1], a new period in investigation of quantum-mechanical phenomena on the macroscopic level began. Namely, a clarification of the notions and planning of experimental situations for observing some physical effects started. The central problem in this respect is a notion of macroscopic differentiation of the states of quantum system whose quantum-mechanical behavior is explored. More precisely, Leggett argues that the term macroscopic quantum-mechanical effect must be related to macroscopically different states, i.e. the system states (and observables) that carry macroscopic properties (and behaviors) of the system as a whole. These states (i.e. observables) must carry classical-physical behavior of the system as well, this posing a task for choosing physical conditions giving rise to observation of typical quantum effects related to these states ${ }^{1}$. Therefore a difference between macroscopic quantum phenomena: the ones usually explored by the methods of (quantum) statistical physics and not related to macroscopically differentiated states (being classified as macroscopic quantum phenomena of the first kind), and those ones regarding macroscopically different (differentiated) states (being classified as macroscopic quantum phenomena of the second kind, and being interesting to us). Numerous different macroscopic quantum phenomena of the second kind, some of them belonging to the fast developing field of the quantum computing and information, unequivocally sharpen the overall problem of universal validity of quantum mechanics.

In the context of macroquantum effects in biophysics, investigations at the end of 20th century in the field of microwave resonance stimulation of acupuncture system, as well as studies of consciousness interactions with microscopic and macroscopic environment - revealed the existence of novel local and nonlocal macroscopic quantum biophysical effects, with far reaching potential implications in medicine, psychology, biology, physics, engineering, philosophy and religion.

\footnotetext{
1 Paradigm of macroscopic, macroscopically differentiated states, are proper states of position (and impulse) of the center of mass of manyparticle system. On the contrary, so called relative coordinates (as observables) neither define macroscopically differentiated states nor carry classical behavior of the system, in any known physical theory or experimental situation.
} 


\section{LOCAL MACROSCOPIC QUANTUM BIOPHYSICAL EFFECTS: MICROWAVE RESONANT STIMULATION OF THE ACUPUNCTURE SYSTEM}

Microwave Resonance Therapy (MRT) is a contemporary technology of the acupuncture stimulation, which represents a synthesis of the ancient Chinese traditional knowledge in medicine (acupuncture) and recent breakthroughs in biophysics: affecting the appropriate acupuncture points by the generation of high frequency microwaves $(52-78 \mathrm{GHz})$, remarkable clinical results in prevention and therapy of stress, as well as of many psychosomatic disorders were achieved [2]. MRT appears under different synonyms: short waves therapy, millimeter therapy, information wave therapy ... The method originates from the former Soviet Union, and first investigations in 1960's, resulted in construction of the first generation of MRT generators, were not related to their medical application but to military industry and satellite communications. Interest for influence of electromagnetic (EM) microwave (MW) radiation upon nonhuman biological objects appeared shortly afterwards (Zalubovskaya in Kharkow [3], Devyatkov et al in Moscow [4]), but necessity for application of MRT upon acupuncture points was discovered only in the early 1980's (Sit'ko et al in Kiev [5]) as appearance of sharply-resonant characteristic eigenfrequencies of human organism - which successfully stimulated development of the second generation of coherent and third generation of noise spectrum MW generators. Sit'ko and collaborators presented the MRT officially in 1989 at the international symposium in Kiev, where international multidisciplinary expert committee headed by Frohlich announced MRT as a perspective approach in the regulation of living systems and MRT clinical results highly efficient, and shortly afterwards the MRT was presented and introduced worldwide.

The quantum-coherent characteristics of MRT therapy should be especially pointed out: (1) highly resonant MW sensory response of the disordered organism sensitive to small changes in the frequency $(0.01-0.1 \%)$ of the manually adapting coherent MRT devices; (2) biologically efficient nontermal MW radiation of the extremely low intensity (up to $10^{-9} \mathrm{~W} / \mathrm{cm}^{2}$ ) and energy (up to $10^{-4}$ $\mathrm{eV}$ ); and (3) neglecting MW energy losses alongside acupuncture meridians $(\sim 1 \mathrm{~m})$. On the basis of these empirical data, Sit'ko and collaborators has proposed that acupuncture system is a macroscopic quantum dynamic structure [5] which might simply be visualized [2,6] as differentiated at the locations of maxima of three-dimensional standing waves, formed as a result of the reflection of coherent MW Frohlich excitations [7] of molecular subunits in the cell membranes, proteins, microtubules etc. This is supported also by other investigations which have demonstrated that differentiation of gap junctions (GJ) channels (of higher density at acupuncture points [8]) - as evolutionary older type of intercell communication, which transmits metabolits and regulatory molecules between nonexcitable cells, and ionic electric signals (presumably by combined classicalionic/quantum-solitonic mechanisms [9]) between excitable cells (of the cardiac muscle, smooth muscles, epithelial liver cells, neurons with electrical synapses, and acupuncture points and meridians) - might be slightly sensitive to voltage [10].

In that context the explanation for efficiency of the MRT, as noninvasive nonmedicamentous medical treatment, should be sought $[2,6]$ : some disorders in the organism give rise to deformation in the standing wave structure of electrical field of the organism in MW region, which influences corresponding changes in spatial structure of the acupuncture system, and consequently its resonant frequencies, resulting in some disease. During the therapy, applying the MW sound at corresponding acupuncture points the excited acupuncture system of the patient is relaxing to the previous healthy condition, while reaching the normal resonant frequencies responses upon the wide spectrum MW source - and following to physiological mechanisms of the acupuncture regulation [11] the organism biochemically overcomes the disease.

The quantum-coherent characteristics of MRT therapy suggest that healthy condition might be considered as an absolute minimum (ground state) of Sit'ko nonlocal selfconsistent macroscopic quantum potential of the organism, while some disorders of an acupuncture system correspond to higher minima of the (spatio-temporally changeable) potential hypersurface in energy-configuration 
space - this being possible explanation of higher MRT sensory responses of the more excited (more disordered) acupuncture system, and poor MRT sensory response of the healthy acupuncture system being already in the ground state $[2,6]$. Such a picture is very close to those of associative neural networks in their energy-configuration spaces $[2,6]$, and pattern recognition as a convergence of the network towards corresponding bottom of the potential hypersurface, as a memory attractor of the neural network $[12,13]$. Thus, the process of psychosomatic disorder might be considered as writing and deepening of the corresponding memory attractor, while the process of MRT therapy might be considered as a subsequent removing of the memory attractor of corresponding psychosomatic disorder - implying that process of the MRT therapy could be most deeply visualized as removal of the very information on corresponding psychosomatic disease [2,6], $\mathrm{cf}$. Fig. 1.

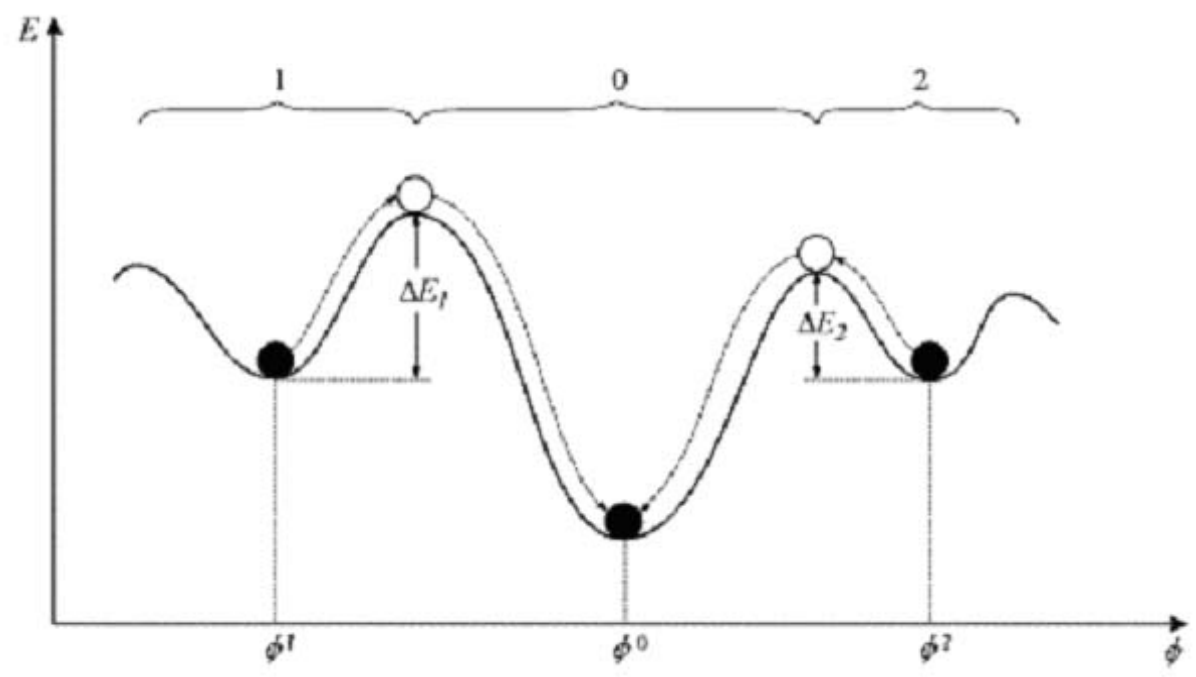

Fig. 1. Schematic presentation of the potential hypersurface of nonlocal selfconsistent macroscopic quantum potential in the acupuncture energy-state space $(E, \phi): 0$ - basin of attraction of the ground (healthy) state $\phi^{0}$; 1 and 2 - basins of attraction of the two excited (psychosomatically disordered) states $\phi^{1}$ and $\phi^{2}$ (hundreds of them being in general). It should be poined out that energy surface changes gradually during the MRT treatment, by decreasing the potential barriers $\Delta E_{1}$ and $\Delta E_{2}$ (and corresponding MRT sensory responses) of the potential wells 1 or 2 , to be overcome in traversing to the 0 basin of attraction of the ground (healthy) state, when organism gradually recovers by further spontaneous approaching the healthy state $\phi^{0}$ (with poor MRT sensory response, as already being in the ground-state). Such a picture is very close to the associative neural networks ones in their energy-configuration spaces, and to pattern recognition as convergence of the neural networks to the bottoms of the potential hypersurfaces, being the attractors of neural networks memory patterns $\phi^{0}, \phi^{1}, \phi^{2}$.

Such a similarity between the two approaches is presumably not a coincidence, as (real) mathematical formalism of the model of Hopfield's associative neural network [12] is analogous [13] to (complex) mathematical formalism of Feynman's propagator version of the Schrodinger equation [14], cf. Appendix I. This also supports the EM/ionic MW/ULF quantum-holographic function of the acupuncture system (like complex oscillatory holographic Hopfield-like neural network [13]), and its essential relation to (complex-valued quantum relativistic) consciousness, as strongly suggested from modeling of altered and transitional states of consciousness [2,6,15], cf. Appendix II (it should be added that the displaced part of ionic acupuncture structure in these states would have a function of an "optical" MW sensor, which can extrasensory perceive an environment, as reported by patients reanimated from clinical death [16]). An additional support that acupuncture system is really related to consciousness is provided by novel meridian (psychoenergetic) therapies (with very fast removing of persistent phobias, alergies and other psychosomatic disorders [17]), whose simultaneous effects of visualization and tapping/touching acupuncture points might be interpreted as a "smearing" of memory attractors of the psychosomatic disorders [17]), through successive imposing new boundary conditions in the acupuncture energy-state space during visualizations of the psychosomatic problems [18]. 
So, it can be said that beside brain's hierarchical electrochemical neural network there exists acupuncture electromagnetic/ionic microwave ultralowfrequency-modulated quantum-holographic electrooptical neural network, with brainwaves being both the interface between them and ULF modulating factor of MW acupuncture network, implying also biophysical basis of psychosomatic disorders i.e. influence of psyche on the body; at the same time this presents explanation for the sensitivity of the organism on the influences of extremely weak external MW/ULF EM fields [19], through $M W / U L F$ EM induction within EM/ionic circulatory acupuncture system, thus modulating acupuncture currents by external EM fields, without any limitations by threshold potentials which do not even exist within gap junction electrical synapses of the acupuncture system.

Such brain/brainwaves/acupuncture system interface is continuously rewriting information from brain hierarchical neural network into acupuncture system, they being there superposed keeping their quantum coherence of the parallel processed subliminal ULF lower-frequency unconscious information within acupuncture EM/ionic MW ULF-modulated quantum-holographic neural network [15]. The process of collapse of the wave function of macroscopic state of the acupuncture system, i.e. final quantum decoherence within the brain, might proceed through the same interface acupuncture system/brainwaves/brain. This is presumably realized through the brain's frontolimbic process [20] of the selection and amplification of the one out of many parallel processed subliminal ULF lower-frequency unconscious information (within the acupuncture system) toward ULF higher-frequency conscious thought in normal states of consciousness (within the brain), being subsequently rewritten into acupuncture system through the interface brain/brainwaves/acupuncture system, thus becoming amplified ULF higher-frequency normal conscious content [15].

\section{NONLOCAL MACROSCOPIC QUANTUM BIOPHYSICAL EFFECTS: INTERACTIONS OF CONSCIOUSNESS WITH MICROSCOPIC AND MACROSCOPIC ENVIRONMENT}

Consciousness is one of the oldest scientific problems, recognized already in ancient times, both in the civilizations of East and West. Although consciousness has been the central theme of philosophical essays for a long time from the very beginning of philosophical thought, or traditional esoteric practices of the East and West which have reached significant level in control of altered states of consciousness with significant philosophical and religious implications - the first scientific attempts to enlighten the phenomenon of consciousness appeared only in psychology of the second half of 19th century (through development of psychophysics and theories of personality), physics of the beginning of 20th century (through development of quantum mechanics and articulation of the problem of the so called wave function collapse, and the role of observer in this process), and computer sciences of the second part of 20th century (through development of artificial intelligence and the conception that the whole cognitive process can be reduced to a computer algorithm). However, due to scientific methodological difficulties the problem of consciousness has not gotten adequate treatment afterwards (corresponding multidisciplinary surveys on the problem of consciousness can be found in monographs of Baars [20], Poper and Eccles [21], Crick [22], Penrose [23], Tart [24], as well as in our proceedings [25]). Nevertheless, scientific investigation of consciousness underwent great renaissance in the last decade of 20th century, with a tendency to become the most significant scientific problem in a near future, being absolutely deserved owing to its unparallel potential implications in many fields (medicine, psychology, biology, physics, engineering, philosophy and religion), as recapitulated in the conclusion of the paper.

Holistic manifestations of consciousness, like altered and transitional states of consciousness [15], conscious/unconscious transitions, consciousness pervading body, and free will [26], as well as nonlocallity of quantum mechanics demonstrated by Einstein-Podolsky-Rosen effect [27] and Bell inequalities multiply confirmed experimentally last years [28], imply that some manifestations 
of consciousness must have deeper quantum origin (in spite of recent Tegmark's theoretical investigations [29] which demonstrate that interactions with environment destroy in less than $10^{-13} \mathrm{~s}$ macroscopic quantum coherence within the brain, whose electrochemical neural networks might correspondingly be sufficient for modeling normal states of consciousness [20-22] - but not altered and transitional states of consciousness as well [24,25], whose nonlocal properties might be understood better through quantum-relativistic manifestations of the acupuncture EM/ionic microwave ultralowfrequency-modulated quantum-holographic neural networks [15], cf. Appendix II).

There are numerous macroquantum models of consciousness (some of them cited in ref. [29], whose aim is to investigate existence of the relevant brain/body degrees of freedom isolated enough to preserve their quantum coherence), and it is difficult to pretend on complete survey of these attempts. Therefore, in this paper we shall restrict ourselves on the approach and critique of the Copenhagen and von Neumann interpretations of quantum mechanics, and those attempts including ours to resolve manifestly open quantummechanical problems in the context of consciousness: (1) physical nature of the wave function nonlinear collapse; (2) physical nature of the instantaneous action on distance of the wave function nonlocal collapse; and (3) existence of the relevant acupuncture macroscopic quantum degrees of freedom within the body (related to altered and transitional states of consciousness) isolated enough to preserve their quantum coherence.

The first attempts to treat consciousness strictly quantummechanically originate from von Neumann [30], being supported by Wigner [31] and lately by Stapp [32]: in contrast to Copenhagen interpretation of Bohr, Dirac, and Heisenberg [33], which insists on positivistic limitations of quantum theory only on the observer knowledge about quantum system (thus becoming essentially subjective and epistemologic theory, as the only reality of this theory is 'our knowledge') - von Neumann's theory offers ontologically objective description of the reality and thus quantum framework for cosmological and biological evolution, including consciousness itself.

Besides, in the problem of quantum measurement i.e. collapse of the initial state of the quantum system in one of the possible probabilistic states of the system (when (linear) Schrodinger equation is a priori suspended and its boundary conditions are replaced by those corresponding to the collapsed state of the quantum system), in contrast to Copenhagen interpretation which treats it (quantum inconsistently) as an ad hoc process in the interaction of quantum system with classical measuring device - von Neumann's theory treats the measuring device quantum consistently by postponing the collapse of the initial quantum state until the final interaction with observer's consciousness in the chain quantum system/measuring device/observer, but also not explaining (nonlinear) nature of the collapse, with additional problem that ontological nonlocality of the collapse requests (relativistically inconsistent) instantaneous action on distance along space-like surfaces (which is the problem also implicitly transferred in the relativistic quantum field theory in spite of the relativistic invariance of the Tomonaga-Schwinger space-like surfaces [32])!

It is obvious that in von Neumann's ontological quantum theory, which consistently introduces phenomenon of consciousness, two open problems still exist: physical nature of the (nonlinear) collapse and instantaneous action on distance of the (nonlocal) collapse.

One possible solution of the problem of (nonlinear) collapse is proposed by Penrose [23] in gravitationally induced reduction of the wave function, in which the gravitational field of measuring device included in the superposition of corresponding possible probabilistic states of the device implies the superposition of different space-time geometries, and when the geometries become sufficiently different (on the Planck-Wheeler scale $\sim 10^{-35} \mathrm{~m}$ ) this implies the break of standard quantum superposition of the state quantum system/measuring device (ill-defined in strictly separate space-time geometries) and Nature must choose between one of them and actually affects objective reduction of the wave function. (While regarding nonalgorithmic quantumgravitational aspects of consciousness [23], Penrose tried to search for sufficiently isolated relevant macroscopic quantum degrees of freedom in microtubular citoskeletal structures of neurons (but not of acupuncture system!), which was undergone to severe critiques in Tegmark's paper [29].) 
According to Raković's biophysical quantum-relativistic model of consciousness [15] (cf. Appendix II), the similar objective reduction of the wave function might have quantumgravitational origin in space-time microscopic wormhole tunnels of highly noninertial microparticle interactions in quantum measurement-like situations (fully equivalent, according to Einstein's principle of equivalence, to strong gravitational fields - in which opening of wormholes is predicted [34]). The question how it is possible that these highly noninertial microparticle processes with inevitable relativistic generation of microparticles' wormholes were not taken into account within quantum mechanics which is yet extremely accurate theory(?) - might be answered as they were(!), but implicitly within the ad hoc von Neumann's projection postulate [30] to account for quantum mechanical "wave packet collapse" in quantum measurement situations (implying also that von Neumann's ad hoc projection postulate is based on quantum gravitational phenomena, being on deeper physical level than nonrelativistic quantum mechanical ones!). On the other hand, nonlocality of collective consciousness [15], as a giant space-time associative neural network with distributed individual consciousnesses (related to bodily acupuncture EM/ionic microwave ultralowfrequently-modulated quantum-holographic neural networks [6], mutually interacting quantum-gravitationally in transitional states of individual consciousnesses [15]), might explain (apparently) instantaneous action on distance in (nonlinear) quantum-gravitationally induced and (nonlocally) channeled collapse by collective consciousness (this being also in accordance with nonlocal Princetone's PEAR experiments of consciousness/machine interactions [35], nonlocal Ditron's experiments of consciousness/nonliving \& living systems' interactions [36], nonlocal Novosibirsk's experiments of consciousness/living \& nonliving systems' interactions [37], as well as with nonlocal clinical Duke's experiments on prayer-accelerated post-surgical patients' recovery [38])!

That quantum mechanically consistent approach to consciousness could really imply nonlocal properties of consciousness, might be supported by our considerations from two additional directions [39]: decoherence theory and cosmology.

Theory of decoherence seeks for physically consistent transition from quantum probabilistic indeterminism to classical reality, in the framework of quantum theory of measurement [40-42], by treating a quantum system as open (but with assumption of universality of the linear Schrodinger equation, the problem of "wave packet collapse" remains unresolved within this approach too [43]). Dugić recently demonstrated the existence of necessary conditions for decoherence process [44], giving rise in macroscopic context to wider physical picture which naturally encounters consciousness (cf. Appendix III): namely, defining of the open quantum system (S) and its environment (E) which macroscopically includes consciousness as well, is the simultaneous process - so that different macroscopic parts of the Universe as the open quantum system (S) are defined simultaneously with its macroscopic environment (E) which includes complementary consciousness (i.e. different macroscopic parts of the observed Universe define different complementary observing consciousness). In this way, within the context of universality of quantum mechanics consciousness is only relative concept, nonlocally defined with all distant parts of the existing observed Universe (although the reverse also holds true!).

This relative metatheory of consciousness is a part of psycho-physical parallelism which emphasizes holistic aspect of the whole Universe and naturally includes consciousness as its part [45-47]. In their lucid and instructive analysis of the collapse problem in quantum mechanics, Barrow and Tipler [47] offer, apart from unattractive solipsism and Everett's "no collapse" theory (with bizarre branching of the system and environment into many copies with different states, continuing their independent existences [48]), another three alternatives: either any being with consciousness can collapse the wave packet by observations, or a "community" of such beings can collectively collapse it, or there is some sort of "ultimate Observer" who is responsible for the collapse - the last two alternatives being natural candidates for incorporation within our relative metatheory of consciousness, thus supporting nonlocallity of (collective) consciousness. Such a viewpoint that macroscopic parts of the Universe play central role in physical understanding of consciousness may not be so surprising, especially if one takes seriously numerous anthropic 
"coincidences" in both classical and quantum cosmology [47,49], with fine-tuning of the model physical parameters in the cosmological initial conditions close to the Big Bang singularity in order that life and consciousness be possible, as well as Ćirković's arguments on relationship of quantum gravitation and transitional states of consciousness through their fundamental non-unitarity (cf. Appendix IV).

\section{CONCLUSION}

It is shown that investigations in the field of microwave resonance stimulation of the acupuncture system, as well as the research of consciousness interaction with microscopic and macroscopic environment - imply the existence of local and nonlocal macroscopic quantum biophysical effects, with great potential implications in many fields:

(1) medicine (brain/consciousness relationship; brainwaves and brain functioning; acupuncture system and cognitive aspects of altered states of consciousness; relationship of nervous system and acupuncture system, and morphogenesis and psychosomatic disorders; local quantum holistic aspects of psychosomatics and wider application of the corresponding microwave resonance therapy on the level of acupuncture system; nonlocal quantum holistic aspects of psychosomatics and wider application of the corresponding transpersonal psychotherapy on the level of individual and collective consciousness);

(2) psychology (mechanism and role of dreaming and meditation, as altered states of consciousness, in the integration of personality; mechanism of ascending of unconscious conflict to conscious level, and interaction of conscious and unconscious in different psychotherapeutic approaches; control of creativity and transpersonal phenomena in transitional states of consciousness, and psychotherapeutic implications for transpersonal clinical psychology);

(3) biology (nonlocal interactions and adaptive mechanisms on the level of species; fundamental understanding of the significance of morality within human population);

(4) physics (fundamental role of individual and collective consciousness in quantum theory of measurement; revealing of deep relationship of consciousness, space-time and structure of matter, in transitional states of individual and collective consciousness; role of transitional states of individual and collective consciousness in controling exits of quantum-gravitational space-time tunnels);

(5) engineering (exploitation of technology of quantum-gravitational tunnels and technology of transitional states of consciousness, for radical surpassing of space-time barriers in transport and communication; computers with artificial consciousness and creativity, on deeper quantumgravitational principles);

(6) philosophy/religion (ontological nature of individual and collective consciousness; removal of the matter/spirit duality; post-mortem consciousness continuity; nonlocal transfer of individual loads on descendants in emotionally loaded transitional states of consciousness; origin of free will; fundamental significance of nonlocal holistic space-time reprogramming of collective consciouness in prayer for fellow-men, enemies, and the dead; renaissance of the behavior based on hesychastic spiritual principles of charity).

\section{APPENDIX I: ON PARALLELS BETWEEN CLASSICAL AND QUANTUM HOPFIELD-LIKE NEURAL NETWORKS}

Hopfield's classical neural networks [12] has been extensively studied, simulated and used in cognitive neurosciences too [50]. However, it has also been shown recently that Hopfield's classical neural network model is mathematically analogous to Feynman's propagator version of the 
quantum theory [14]. Hence, in this appendix the formal informational parallelism between classical and quantum Hopfield-like neural networks will be overviewed.

In the model of Hopfield's classical neural network, emergent collective computation is regulated by minimization of the Hamiltonian energy function [12]

$$
H=-\frac{1}{2} \sum_{i=1}^{N} \sum_{j=1}^{N} J_{i j} q_{i} q_{j}=-\frac{1}{2} \sum_{i=1}^{N} \sum_{j=1}^{N} \sum_{k=1}^{P} q_{i}^{k} q_{j}^{k} q_{i} q_{j}
$$

where $q_{i}$ is the actual collective activity of the $i$ th neuron (out of $N$ existing neurons in the network), while $q_{i}^{k}$ is the contribution of the activity of the neuron $i$ when taking part in encoding the kth memory pattern (out of $P$ existing memory patterns in the neural network). The process of gradientdescent of this energy function is a result of a web of interactions between the system of neurons described by vector $\boldsymbol{q}$ (with elements $q_{i}$ ) and the system of synaptic connections, described by the memory matrix $\boldsymbol{J}$ (with elements $J_{i j}$ ).

Thus, in the model of Hopfield classical neural network, Hebbian dynamic equation for neuronal activities

$$
q_{i}\left(t_{2}=t_{1}+\delta t\right)=\sum_{j=1}^{N} J_{i j} q_{j}\left(t_{1}\right) \quad \text { or } \quad \boldsymbol{q}_{\text {out }}\left(t_{2}\right)=\boldsymbol{J} \boldsymbol{q}_{\text {in }}\left(t_{1}\right)
$$

and dynamic equation for synaptic connections (weights)

$$
J_{i j}=\sum_{k=1}^{P} q_{i}^{k} q_{j}^{k} \quad \text { or } \quad \boldsymbol{J}=\sum_{k=1}^{P} \boldsymbol{q}^{\boldsymbol{k}} \boldsymbol{q}^{\boldsymbol{k} \boldsymbol{T}}
$$

exhibit a joint classical parallel-distributed information processing system. This is one of the simplest algorithms useful for theoretical brain modeling [50].

Equation (I.1) is global (variational) description, while system of equations (I.2-3) is local (interactional) description of the learning of input-data vectors $\boldsymbol{q}^{\boldsymbol{k}}$, in Hopfield's classical neural network. The corresponding neuronal activities can be inserted in the system of neurons $\boldsymbol{q}$ iteratively, or can be put in the very beginning simultaneously into the Hebb memory matrix $\boldsymbol{J}$ which contains all synaptic weights $J_{i j}$.

Equations (I.2-3) can be rewritten in continuous form incorporating spatio-temporal description of neuronal and synaptic activities:

$$
\begin{gathered}
\boldsymbol{q}_{\text {out }}\left(\boldsymbol{r}_{2}, t_{2}\right)=\iint \boldsymbol{J}\left(\boldsymbol{r}_{2}, t_{2}, \boldsymbol{r}_{\mathbf{1}}, t_{1}\right) \boldsymbol{q}_{\text {in }}\left(\boldsymbol{r}_{\mathbf{1}}, t_{\mathbf{1}}\right) \boldsymbol{d} \boldsymbol{r}_{1} d t_{1} \\
\boldsymbol{J}\left(\boldsymbol{r}_{2}, t_{2}, \boldsymbol{r}_{\mathbf{1}}, t_{1}\right)=\sum_{k=1}^{P} \boldsymbol{q}^{\boldsymbol{k}}\left(\boldsymbol{r}_{2}, t_{2}\right) \boldsymbol{q}^{\boldsymbol{k} \boldsymbol{T}}\left(\boldsymbol{r}_{\mathbf{1}}, t_{1}\right) \quad \text { ili } \quad \boldsymbol{J}\left(\boldsymbol{r}_{2}, \boldsymbol{r}_{\mathbf{1}}\right)=\sum_{k=1}^{P} \boldsymbol{q}^{\boldsymbol{k}}\left(\boldsymbol{r}_{2}\right) \boldsymbol{q}^{\boldsymbol{k} \boldsymbol{T}}\left(\boldsymbol{r}_{\mathbf{1}}\right)
\end{gathered}
$$

The memory recall in Hopfield's classical neural network is done by input-output transformation $\boldsymbol{q}_{\text {out }}=\boldsymbol{J} \boldsymbol{q}_{\text {in }}$, or in developed form

$$
\boldsymbol{q}_{\text {out }}\left(\boldsymbol{r}_{2}, t_{2}=t_{1}+\delta t\right)=\int \boldsymbol{J}\left(\boldsymbol{r}_{2}, \boldsymbol{r}_{\mathbf{1}}\right) \boldsymbol{q}_{\text {in }}\left(\boldsymbol{r}_{\mathbf{1}}, t_{1}\right) d \boldsymbol{r}_{\mathbf{1}}=\int\left[\sum_{k=1}^{P} \boldsymbol{q}^{\mathbf{k}}\left(\boldsymbol{r}_{2}\right) \boldsymbol{q}^{\mathbf{k} \boldsymbol{T}}\left(\boldsymbol{r}_{\mathbf{1}}\right)\right] \boldsymbol{q}_{\text {in }}\left(\boldsymbol{r}_{\mathbf{1}}, t_{1}\right) d \boldsymbol{r}_{\mathbf{1}}
$$

From equation (I.6) it is obvious that if the input vector $\boldsymbol{q}_{\boldsymbol{i n}}$ is most similar to some of the previously memorized vectors, say $\boldsymbol{q}^{\mathbf{1}}$ (and simultaneously almost orthogonal to all other memory vectors $\boldsymbol{q}^{\boldsymbol{k}}, k$ 
$\neq 1$ ), then the output vector $\boldsymbol{q}_{\text {out }}$ converges to the memory pattern-qua-attractor $\boldsymbol{q}^{\mathbf{1}}$, i.e. Hopfield's classical neural network associatively recognizes the vector $\mathbf{q}$.

Haken has shown that introduction of biologically more plausible neuronal oscillatory activities gives richer dynamics of the neural network [51], with Hopfield's classical neural net realvalued variables replaced by the complex-valued ones (similarly to quantum variables, although in contrast to thus conveniently modified classical formalism, the complex-valued quantum formalism is essential). A step further is done with quantum generalization of Hopfield's neural network: Sutherland's holographic neural network [52] and, equivalent to it, Peruš's model of Hopfield-like quantum neural network [13]. In this appendix we shall review Peruš's model, based on the direct mathematical correspondence between classical neural (left) and quantum variables (right) and corresponding Hopfield-like classical and quantum equations, respectively:

$$
\begin{gathered}
\boldsymbol{q} \Leftrightarrow \phi, \quad \boldsymbol{q}^{k} \Leftrightarrow \phi^{k}, \quad \boldsymbol{J} \Leftrightarrow G \\
(\text { I.4) } \Leftrightarrow(\text { I.7), (I.5) } \Leftrightarrow \text { (I.8), (I.6) } \Leftrightarrow \text { (I.9) }
\end{gathered}
$$

The equations in pairs are mathematically equivalent, implying similar collective dynamics of the neural and quantum systems, in spite of different nature of the set of neurons $(\boldsymbol{q})$ and their memory synaptic connections $(\boldsymbol{J})$ in the neural network, on the one hand, and wave functions $(\phi)$ and their propagator connections $(G)$ in the quantum system, on the other hand.

So, in Peruš's model of the Hopfield-like quantum neural network [12], dynamic equation for the wave function of the quantum state

$$
\phi_{\text {out }}\left(\boldsymbol{r}_{2}, t_{2}\right)=\iint G\left(\boldsymbol{r}_{2}, t_{2}, \boldsymbol{r}_{1}, t_{1}\right) \phi_{\text {in }}\left(\boldsymbol{r}_{1}, t_{1}\right) d \boldsymbol{r}_{1} d t_{1} \text { or } \phi_{\text {out }}\left(t_{2}\right)=G \phi_{\text {in }}\left(t_{1}\right)
$$

and dynamic equation for the propagator of the quantum system

$$
G\left(\boldsymbol{r}_{2}, t_{2}, \boldsymbol{r}_{1}, t_{1}\right)=\sum_{k=1}^{P} \phi^{\boldsymbol{k}}\left(\boldsymbol{r}_{2}, t_{2}\right) \phi^{\boldsymbol{k}}\left(\boldsymbol{r}_{1}, t_{1}\right)^{*} \text { or } G\left(\boldsymbol{r}_{2}, \boldsymbol{r}_{1}\right)=\sum_{k=1}^{P} \phi^{\boldsymbol{k}}\left(\boldsymbol{r}_{2}\right) \phi^{\boldsymbol{k}}\left(\boldsymbol{r}_{1}\right)^{*}
$$

exhibit a joint quantum parallel-distributed information processing system, where $\phi^{k}$ are eigen wave functions of the quantum system. So $\phi^{k}$ represents the memory state, and the propagator $G$ constitutes the memory of thus informationally interpreted quantum system! (Actually, so defined propagator $G$ is related to the usually used Green function propagator $\bar{G}$, by equation $G=-i \bar{G}$ [14].)

The memory recall in Hopfield-like quantum neural network is done by input-output transformation $\phi_{\text {out }}=G \phi_{\text {in }}$, or in developed form

$$
\phi_{\text {out }}\left(\boldsymbol{r}_{2}, t_{2}=t_{1}+\delta t\right)=\int G\left(\boldsymbol{r}_{\mathbf{2}}, \boldsymbol{r}_{\mathbf{1}}\right) \phi_{\text {in }}\left(\boldsymbol{r}_{\mathbf{1}}, t_{1}\right) \boldsymbol{d} \boldsymbol{r}_{\mathbf{1}}=\int\left[\sum_{k=1}^{P} \phi^{\boldsymbol{k}}\left(\boldsymbol{r}_{\mathbf{2}}\right) \phi^{\boldsymbol{k}}\left(\boldsymbol{r}_{\mathbf{1}}\right)^{*}\right] \phi_{\text {in }}\left(\boldsymbol{r}_{\mathbf{1}}, t_{1}\right) \boldsymbol{d} \boldsymbol{r}_{\mathbf{1}}
$$

i.e. in the other form (where one can recognize the quantum superposition principle, i.e. the development of the wave function $\phi_{\text {out }}$ over the wave functions $\phi^{k}$ )

$$
\phi_{\text {out }}(\boldsymbol{r}, t)=\sum_{k=1}^{P} c^{\boldsymbol{k}}(t) \phi^{\boldsymbol{k}}(\boldsymbol{r})=\sum_{k=1}^{P} \int\left[\phi^{\boldsymbol{k}}(\boldsymbol{r})^{*} \phi_{\text {in }}(\boldsymbol{r}, t) \boldsymbol{d} \boldsymbol{r}\right] \phi^{\boldsymbol{k}}(\boldsymbol{r})
$$


From equations (I.9) and (I.9') it is obvious that if the input wave function $\phi_{\text {in }}$ is most similar to some of the previously memorized wave function, say $\phi^{l}$ (and simultaneously almost orthogonal to all other memory eigen wave functions $\phi^{k}, k \neq 1$ ), then the output wave function $\phi_{\text {out }}$ converges to the memory pattern-qua-attractor $\phi^{1}$, i.e. Hopfield-like quantum neural network associatively recognizes the eigen wave function $\phi^{l}$.

Or translated into orthodox language of the quantum physics [14], in the above example the propagator $G$ represents the projector onto the wave subspace/state $\phi^{l}$, i.e. makes reduction (collapse) of the wave function $\phi_{\text {in }}$ of the quantum system into the eigen state $\phi^{l}$. Naturally, the collapse of the wave function of the quantum processor (not only of the hereby considered associative quantum memory) is also the final phase of the quantum computers as a network of quantum neurons (qubits) [53] - as well as the quantum decoherence within the brain [29], presumably through the brain frontolimbic [20,21] process of the selection and amplification of the one out of many (parallel processed subliminal ULF lowerfrequancy) unconscious pieces of information toward (ULF higherfrequancy) conscious thought in normal states of consciousness [54].

Hopfield-like quantum neural networks are better then the classical ones because of the quantum phase differences which improve classical Hebbian amplitude coding [50]. Namely, by insertion of the eigen wave functions $\phi^{k}$ in the form of modulated plane waves or wavelets [13],

$$
\phi^{\boldsymbol{k}}(\boldsymbol{r}, t)=A_{k}(\boldsymbol{r}, t) e^{\frac{i}{\hbar} S_{k}(r, t)}
$$

the propagator of the quantum system (I.8) becomes

$$
G\left(\boldsymbol{r}_{2}, t_{2}, \boldsymbol{r}_{1}, t_{1}\right)=\sum_{k=1}^{P} A_{k}\left(\boldsymbol{r}_{2}, t_{2}\right) A_{k}\left(\boldsymbol{r}_{1}, t_{1}\right) e^{\frac{i}{\hbar}\left(S_{k}\left(r_{2}, t_{2}\right)-S_{k}\left(r_{1}, t_{1}\right)\right)}
$$

which describes the two-fold memory encoding: through the amplitude correlations, similarly to Hebbian rule in classical associative neural networks [50],

$$
\sum_{k=1}^{P} A_{k}\left(\boldsymbol{r}_{2}, t_{2}\right) A_{k}\left(\boldsymbol{r}_{1}, t_{1}\right)
$$

and through the phase differences, $\delta S_{k}=S_{k}\left(\boldsymbol{r}_{2}, t_{2}\right)-S_{k}\left(\boldsymbol{r}_{1}, t_{1}\right)$, similarly to holography [52].

The correspondence of the informational-physical laws of neural and quantum physics, presented in this appendix, seems to represent only one of illustrations of the deep interrelations of the laws of Nature on different levels. It has also been shown recently [55] that physical laws which describe simple clocks, simple computers, black holes, space-time foam, and holographic principle are interrelated!

\section{APPENDIX II: ON BIOPHYSICAL QUANTUM-RELATIVISTIC MODEL OF ALTERED AND TRANSITIONAL STATES OF CONSCIOUSNESS}

According to Raković's biophysical quantum-relativistic model of altered states of consciousness [15], dreaming and similar hallucinate states are characteristics of the low-dielectric altered states of consciousness, with relativistic mixing of normally conscious and unconscious contents and extremely dilated subjective time base. Namely, according to this biophysical model, it is postulated that subjective reference system of consciousness is related to EM field of the ionic 
currents in nonhomogeneous parts of the bodily acupuncture ionic structures, and from the biophysical analysis of the models it follows that it must be displaced out of body in altered states of consciousness! On the other hand, additional macroscopic arguments imply that consciousness is related to EM field of the bodily acupuncture EM/ionic microwave ultralowfrequency-modulated quantum-holographic neural network [15], while analogy of the mathematical formalisms [13] of the models of Hopfield's associative neural network [12] and Feynman's propagator version of Schrodinger equation [14] implies that consciousness might be a property of the physical field itself - implying that giant nonlocal quantum neural network with distributed individual consciousness processing units behaves as a collective consciousness of Nature itself, as widely claimed by esoteric/religious traditions $[45,46]$. This model might also provide additional route to the quantumgravitational objective wave-packet reduction, and the role of consciousness in this process [15].

Namely, this might be deeply connected with the role of collective consciousness (as a composite quantum state $\Phi$ of all individual consciousness $\phi^{k}\left(\Phi \sim \prod_{k} \phi^{k}\right)$ in quantum theory of measurement, where collective consciousness with its self-assembling $\Phi \rightarrow \Phi_{i}$ (equivalent to convergence of quantum Feynman's propagator to the state $\Phi_{i}$ ) contributes in instantaneous quantum channeling $\Psi \rightarrow \Psi_{i}$ (reduction of the initial wave function $\Psi$ into one of (possible) probabilistic eigenstates $\Psi_{i}$ ) in quantum measurement-like situations. The very physical mechanism of the objective reduction in (nonlinear) collapse of the wave packet might have quantum-gravitational origin (similar to Penrose's gravitation induced collapse [23]), in the spacetime microscopic wormhole tunnels of highly noninertial microparticle's interactions in quantum measurement-like situations (fully equivalent to extremely strong gravitational fields according to Einstein's principle of equivalence, when relativistic generation of wormholes is predicted [34]).

It should be also pointed out that the above collective consciousness' self-assembling $\Phi \rightarrow \Phi_{i}$ in the quantum theory of measurement (on numerously prepared investigated quantum system $\Psi$ ) should be interpreted as purely probabilistic in Penrose's gravitation-induced objective reduction of the wave packet (with relative frequency of their appearance given by quantum-mechanical probability $\left|a_{i}\right|^{2}$ of realization of corresponding microparticls' eigenstates $\Psi_{i}$, where $\left.\Phi \Psi=\sum_{i} a_{i} \Phi_{i} \Psi_{i}, i=1,2,3, \ldots\right)$, depending not on the previous history of the repeatedly prepared quantum system. However, this might not be the case for biological individual consciousness' selfassembling, being history-dependent deterministic one (resulting in history-ponderous more probable convergence of the macroscopic consciousness-related-acupuncture electromagnetic/ionic microwave ultralowfrequency-modulated oscillatory holographic Hopfield-like associative neural network to the deeper particular attractor in the potential hypersurface [13], or equivalently to more probable convergence of quantum Feynman's propagator to the state $\phi^{k}$, fixedly determined by the more dominant state of individual consciousness), implying that strong preferences in individual futures might exist, governed by individual mental loads, as widely claimed in Eastern tradition [45], also presumably supported by contemporary mathematical discovery of the hidden Old Testament's "Bible code" [56]; the same may apply to collective futures too, governed by interpersonal mental loads [15]. It should be also noted that these preferences in individual and collective futures might be anticipated in transitional states of consciousness [15] which might be the basis of intuition, precognition and deep creative insights [57]. What is really anticipated in transitional states of consciousness of individual consciousness might be the evolved state of cosmic collective consciousness $\Phi(\mathrm{t})$ (to which our individual consciousness $\phi^{k}$ has access, being the constituting part of cosmic collective consciousness), which is quantum-mechanically described by deterministic unitary evolution governed by Schrodinger equation.

However, it also seems that (non-Schrodinger governed) nonlocal individual/collective consciousness reassembling $\left(\Phi \rightarrow \Phi_{i}\right.$ ) is possible, with direct influence on the collapse of the 
observed system ( $\Psi \rightarrow \Psi_{i}$ ), which is supported by Princeton PEAR human/machine experiments [35]. In these experiments (even distant) human operators have been able solely by volition to influence the statistical behaviour of sophisticated machines with (otherwise) strictly random outputs, in a statistically repeatable effects (of the order of a few parts in ten thousand) - but individually not reproducible at any moment, which is a standard request in contemporary scientific experiments! All this can be accounted by intentional transitional transpersonal biological (nonSchrodinger governed) quantum-gravitational tunneling of the operator's individual consciousness with mental addressing on the machine's content of collective consciousness in the operator's transitional states of consciousness (short and therefore not easily reproducible [57] highly noninertial processes of the out-of-body space-time quantum-gravitational mentally-channeled tunneling of operator's part of the EM/ionic acupuncture system [15]), thus channeling intentionally the operator/machine composite state of collective consciousness $\left(\Phi \rightarrow \Phi_{i}\right)$, and automatically influencing the machine output $\left(\Psi \rightarrow \Psi_{i}\right)$ in the non-Schrodinger quantum-gravitationally governed collapse-like process ( $\Phi \Psi \rightarrow \Phi_{i} \Psi_{i}$ ). As a consequence one could really conjecture that consciousness might be essential property of Nature at different structural levels, macroscopic and microscopic, animate and inanimate, being presumably related to the unified field itself $[45,46]$.

That such nonlocal (non-Schrodinger governed) individual/collective consciousness reassembling $\left(\Phi \rightarrow \Phi_{i}\right.$ ) is really possible, can be additionally supported by 1998' Duke feasibility study conducted by the Duke University and Durham Veterans Affairs medical centers, in which angioplasty patients with acute coronary syndromes who were simultaneously prayed for by seven different religious sects around the world did 50 percent to 100 percent better during their hospital stay than patients who were not prayed for by these groups [38]! This is in accordance with longlasting empirical evidences of various Eastern and Western esoteric/religious traditions [45,58]. It should be added, on this traditional line of evidences, that according to the consciousness-relatedacupuncture electromagnetic/ionic microwave ultralowfrequency-modulated oscillatory holographic Hopfield-like associative neural network [15], esoteric/religious notions such as [45,58] psychic body (manomaya, lingasarira, manovijnana, ka, psyche, astral body, subtle body, soul, ...) and spiritual body (vijnanamaya, suksmasarira, manas, ba, thymos, noetic body, mental body, mind, ...) are biophysically inevitably associated with a partly displaceable (from the body, in altered and transitional states of consciousness) ionic acupuncture system, and an EM component of ionic currents embedded within it, respectively; also, the ionic condensations in the structured acupuncture system, with an EM component of ionic currents embedded within it, behave like distributed centers of consciousness (yogic chakras); finally, the ionic nature of the etheric vitality (qi, prana, pneuma, bioenergy, ...) and etheric body (related to nondifferentiated ions, essentially important for many biochemical processes within the body), might be noticed.

Finally, it should be pointed out that theoretical biophysical modeling of cognitive processes [54,59] might have significant psychotherapeutic implications: (1) dreaming and meditation (as a prolonged altered state of consciousness [45]) contribute to integration of conscious and unconscious contents around healthy associative memory ego-state, which results in growth of personality and alleviation of emotional conflicts; (2) autogenic training (as the most contemporary method of medical relaxation $[2,54,60]$ ) helps contextual messages to be associatively memorized at the subconscious level, from where they contribute to softening of undesired psychosomatic reactions on everyday stressful situations; however, for persons with strong psychological conflicts the main priority is reprogramming of their psychological conflicts and our modeling implies that this is possible in one of two possible ways: (3) deep psychotherapeutic practices (psychoanalytic, group, humanistic, gestalt, existential, body, transpersonal, expressive, hypno, integrative, experiential, ... orientation $[61,62]$ ) oriented to recognition of the origin of unconscious conflict and its ascending to conscious level, thus diminishing corresponding unconscious conflicting associative memory state, which enables further integration of personality around healthy associative memory ego-state [54]; (4) transpersonal Christian/religious technique (especially of hesychastic prayer $[58,62]$ ) bears probably essential transpersonal aspect of psychological conflict 
and necessity of its simultaneous transpersonal reprogramming in all persons involved, contributing to its radical and final reprogramming at the level of spatio-temporal associative memory network of collective consciousness, which seems to be only possible through the prayer for others in transitional states of consciousness of the praying person $[15,59]$.

\section{APPENDIX III: ON THE ROOTS OF RELATIVE METATHEORY OF CONSCIOUSNESS IN THE QUANTUM DECOHERENCE THEORY}

The process (effect) of decoherence can be qualitatively defined as a physical process "induced" by environment $\mathrm{E}$ upon the quantum system $\mathrm{S}$, which leads to effective, approximately classical-physical behavior of the quantum system. For manyparticle systems, the effect is enormously quick (say of the order $\tau<<10^{-23} \mathrm{~s}$ ), and hence it is not surprising that this effect is observed only recently, in a very demanding experimental conditions.

The system $\mathrm{S}$ is open quantum system to which unitary (reversible Schredinger) temporal evolution cannot be applied. However, for composite system $\mathrm{S}+\mathrm{E}$ the universal validity of quantum mechanics is supposed, i.e. that it evolves in time according to Schredinger equation, with Hamiltonian $\hat{H}=\hat{H}_{S}+\hat{H}_{E}+\hat{H}_{\text {int }}$ which exists in the unitary operator of temporal evolution of the composite system: $\quad \hat{U}(t)=\exp (-2 \pi i \hat{H} t / h) \cong \exp \left(-2 \pi i \hat{H}_{\text {int }} t / h\right)=\hat{U}_{\text {int }}(t) \quad$ (as the interaction Hamiltonian $\left(\hat{H}_{\text {int }}\right)$ is usually much greater than the "self-Hamiltonian" of the mutually noninteracting system $\left(\hat{H}_{S}\right)$ and environment $\left.\left(\hat{H}_{E}\right)\right)$. Therefore, the problem of the decoherence theory is to calculate the "density matrix" of the system $\mathrm{S}$, defined as

$$
\hat{\rho}_{S}=\operatorname{tr}_{E}\left(\hat{U}_{\text {int }}(t)|\Psi(t=0)\rangle_{S E S E}\langle\Psi(t=0)| \hat{U}^{*}{ }_{\text {int }}\right)
$$

where " $t r_{E}$ " denotes integration over degrees of freedom of the environment, while $|\Psi(t=0)\rangle_{S E}$ represents the initial state of the composite system $\mathrm{S}+\mathrm{E}$ - alongside with finding ortonormalized basis $\left.\{i\rangle_{S}\right\}$ in the Hilbert state space of the system $\mathrm{S}$, where at least approximate diagonal form of the density matrix (III.1) is obtained: $\rho_{S i i^{\prime}} \equiv_{S}\left\langle i^{\prime}\left|\hat{\rho}_{S}\right| i\right\rangle_{S} \approx 0, i \neq i^{\prime}$.

In a part of the literature the effect of decoherence is proclaimed as a very basis of boarderline between the two quantum mechanical systems - the (open) system and its environment - in such a way that if there is no decoherence, then there is no boarder-line between the two systems mentioned above. Recent discovery of the existance of necessary conditions for decoherence [44] implies that quantum mechanics offers much more than one can (classically) comprehend and interpret. However, in the context of the above interpretation (according to which if there is no decoherence then there is no boarder-line between physical systems) the existence of necessary conditions poses the question: what about, mathematically possible, no-decoherence situations, when there is no possibility to establish boarder-line between the (open) quantum system and its (quantum) environment? At first sight, there is nothing to add here. However, it is not so; even more, a new "horizon" in investigation of the deeper fundamentals of the process of decoherence is opened, leading basically to some sort of metaphysical theory of consciousness. Some of the results will be shortly presented bellow.

Let the two (quantum) systems, $\mathrm{S}_{1}$ and $\mathrm{E}_{1}$, are given with their "degrees of freedom", alongside with their interaction which prevents the process of decoherence [44]. Then the following reasoning appears possible and physically justified: let us introduce new coordinates, i.e. define two new systems (new system and its environment), $\mathrm{S}_{2}$ and $\mathrm{E}_{2}$, but in such a way that degrees of freedom of these systems represent (analytical) functions of degrees of freedom of the both "old" 
ones, $\mathrm{S}_{1}$ and $\mathrm{E}_{1}$, while the same interaction (i.e. the same interaction Hamiltonian), expressed in terms of new degrees of freedom, does not prevent decoherence. Then, the decoherence which is forbidden in the "old" composite system $\mathrm{S}_{1}$ and $\mathrm{E}_{1}$, now enables establishment of the boarder-line in the "new" composite system ("appeared" by redefining degrees of freedom in the same whole, $\mathrm{S}_{1}+$ $\left.\mathrm{E}_{1}=\mathrm{S}_{2}+\mathrm{E}_{2}\right)$ ! I.e. the condition for decoherence simultaneously defines both the open system $\left(\mathrm{S}_{2}\right)$ and its environment $\left(\mathrm{E}_{2}\right)$. The "old" system and its environment, according to initial presumption, remain indistinguishable.

These transformations can be formally presented in the following way. Let the open system $\mathrm{S}_{1}$ is given with its degrees of freedom and (its canonically conjugated) generalized momenta, $\left(x_{i}, p_{i}\right)$, and analogously for its environment $\mathrm{E}_{1},\left(X_{j}, P_{j}\right)$. Let the new (open) system and its environment, $\mathrm{S}_{2}$ and $\mathrm{E}_{2}$, are given with their variables, $\left(\xi_{m}, \pi_{m}\right)$ and $\left(\Xi_{n}, \Pi_{n}\right)$, respectively. Then the considered transformations have the form:

$$
\begin{aligned}
& \xi_{m}=\xi_{m}\left(x_{i}, p_{i} ; X_{j}, P_{j}\right) \\
& \pi_{m}=\pi_{m}\left(x_{i}, p_{i} ; X_{j}, P_{j}\right)
\end{aligned}
$$

for every variable (degree of freedom and generalized momentum) of the "new" system $\mathrm{S}_{2}$ (and (analogously) of its environment $\mathrm{E}_{2}$ ). The Eq.(III.2) points out the following: the considered transformations (for instance, linear) interconnect degrees of freedom and generalized momenta of both ("old") system $\mathrm{S}_{1}$ and ("old") environment $\mathrm{E}_{1}$. So, the coordinates of the "new" system $\mathrm{S}_{2}$ (and, simultaneously, of its environment $\mathrm{E}_{2}$ ) are functions of the variables of both "old" systems, $\mathrm{S}_{1}$ and $\mathrm{E}_{1}$. In the quantum mechanical formalism, these transformations bear one very important consequence. Namely, in order that measurement of observables of the "old" systems might be possible, the measurement of observables of the "new" systems must be possible - simultaneously. But, this is not possible due to non-commuting degrees of freedom and momenta (of the "new" systems), and hence the transformation (III.2) is qualitatively different from the analogous classical transformations: if the interaction Hamiltonian is such that, in respect to degrees of freedom of the "new" systems, allows decoherence, then the decoherence concerns "new" systems, while (due to no-decoherence in respect to "old" systems) the "old" systems remain undistinguishable. Or more precisely: degrees of freedom of the "old" systems $\left(\mathrm{S}_{1}, \mathrm{E}_{1}\right)$ are not defined by the condition of decoherence, while because of the uncertainty relations they cannot be measured even indirectly by virtue of measurement of observables of the "new" systems $\left(\mathrm{S}_{2}, \mathrm{E}_{2}\right)$. This is the essence of the term "undistinguishable" for the "old" systems.

The generalization of this reasoning on the whole macroscopic systems of the physical Universe leads to an interesting conclusion. Namely, in the isolated macroscopic part of the Universe (MPU) the local transitions of the type $\mathrm{S}_{1}+\mathrm{E}_{1} \rightarrow \mathrm{S}_{2}+\mathrm{E}_{2}$ are forbidden, i.e. once established boarder-line (criterion for decoherence) cannot be changed locally, neither spontaneously nor by any external action. While eventual global transition, regarding MPU as a whole, is unobservable. The related physical argument is following: the physical basis of consciousness is, by presumption, manyparticle system which is subsystem of MPU. Hence the imagined global transition of the above type simultaneously concerns this physical system too, and therefore the basis for observation of the considered global transition disappears. In other words: the above mentioned transformations of the degrees of freedom redefine the (open) quantum systems, their environments, but also physical basis of consciousness, giving rise that, effectively, consciousness becomes a relative notion - defined by degrees of freedom of other systems (and their environments). Therefore, the considered global transition defines MPU as a whole, including definition of (physical basis) of consciousness. As different definitions of MPU are mutually exclusive (at least in the context of existing paradigms), it is possible to talk about relative metaphysical theory of consciousness, which bears some sort of (but not in the sense of von Neumann's theory) psycho-physical parallelism. 


\section{APPENDIX IV: ENTROPY AND NONUNITARITY IN THE PHYSICS OF BLACK HOLES AND CONSCIOUSNESS}

There are at least two aspects of the contemporary physics of black holes and its application in cosmology which imply holistic nature of the universe, and have significance for any anthropic way of thinking. The first one is related to the fact that black holes, considered as classical objects with some quantum properties, have enormous number of degrees of freedom manifested through entropy of gravitating matter. This is represented by Bekenstein-Hawking formula $[63,64]$ :

$$
S_{B H}=\frac{k c^{3}}{G \hbar} \frac{A}{4}
$$

where $A$ is the surface of the black hole's event horizon, and the rest are fundamental physical constants ( $k$ is the Boltcmann constant, $c$ is the velocity of light in vacuum, $G$ is the Newton gravitational constant, and $\hbar$ is the Planck constant divided by $\pi$ ). It is obvious that the entropy of matter inside the event horizon is proportional to the surface of the horizon, while the physical problem of the (singular or nonsingular) state of the matter is not important from this point of view. In the simplest case of the spherically-symmetric nonrotating and electrically neutral black hole, the horizon is the sphere of the surface $A=4 \pi R^{2}$, where $R$ is the Schwartzshild (gravitation) radius given by $R=2 \mathrm{Gm} / \mathrm{c}^{2}$ (where $m$ is the mass of the black hole). In combination with (IV.1) this gives

$$
S_{B H}=\frac{2 \pi G k}{\hbar c} m^{2}
$$

Now it is possible to calculate gravitational entropy of the matter inside our cosmological (particle) horizon, if it would collapse in a some way into giant black hole. This would be the state of maximal entropy, as the state of the matter within black hole, as shown by Bekenstein, is the most probable one from the viewpoint of the gravitational interaction. Any other state, like the one observed today with the matter mainly concentrated in galaxies, stars, planets, etc. with a small number of black holes, is a priori less probable. It should be noted, on the other hand, in the context of contemporary cosmological models, that situation when all the matter was within its gravitational radius actually took part in the past: all the matter inside our horizon was in the initial singularity of the Big Bang about 14 billion years ago.

By applying the Boltzmann formula $S=k \ln W$, it is possible to estimate the statistical weight $(W)$ of the state characterized by the entropy $(S)$ of the present universe (much less than maximal entropy from the Eq.(IV.2) estimated by Penrose (1989) as $\sim 10^{123}$, in the units of the Boltzmann constant $k$ [23]). It is already qualitatively obvious that the presence of the logarithm in the Boltzmann equation implies that the present state is extremely less probable; its probability $\left(W^{-1}\right)$ is of the order $\exp \left(-10^{123}\right)$ !!! Such a small number cannot be found in any other place in natural sciences. Hence this is most spectacular example of the "fine tuning", i.e. anthropic coincidences in cosmology. The former originates from the fact, realized by astrophysicists long ago, that very low gravitational entropy (sinonimous for relatively high uniformity of the early universe) is necessary for creation of the galaxies, which is the condition for existence of biological systems and intelligent observers. This conclusion was explicitly formulated by Collins and Hawking [65] even before Bekenstein's papers and sudden increase of interest for the problem of the gravitational entropy.

The second aspect of the physics of black holes, of the great importance for problems of consciousness and intelligent observers, is the possibility of essential violation of determinism in semiclassical process of the evaporation of black holes (Hawking's process). The hypothesis that 
transitional states of consciousness include inherently indeterministic evolution ("free will") can be potentially explained physically as a residuum of deeper physical processes that include nonunitarity. Such processes were seriously suggested by now only in the context of quantum gravitation, the Hawking process precisely. This argument deserves more detailed consideration, as it is less probable that this is merely coincidence. Rather, it presents an indirect support for the hypothesis of significant quantum gravitational role in the functioning of consciousness.

Hawking (1982) was the first to point on the possibility of nonunitary evolution of a physical system, as one of possible answers to the problem of the loss of information in the black holes [66]. Namely, let us consider pure quantum state $|\psi\rangle$ which corresponds to the distribution of matter of the mass $m$ which collapses under the influence of self-gravitation. The density matrix of such state is given by $\rho=|\psi\rangle\langle\psi|$, with vanishing entropy $S=-\operatorname{tr} \rho \ln \rho$. If $m$ is large enough, the matter will create the black hole inevitably. Afterwards, the black hole will evaporate slowly through the Hawking process, by emitting the black body radiation (which does not carry any nontrivial information). The semiclassical procedure used by Hawking in derivation of formula for the temperature of black hole will not be applicable when the mass of the evaporating black hole approaches to Planck mass, but what will happen with the information about the initial state still enclosed within the black hole? This is a puzzle of the loss of information within black holes (cf. for instance [67]).

The possibility proposed firstly by Hawking is that the black hole simply evaporates completely, so that information is lost irreversibly. This might be described as a fundamental unpredictability of black holes (and analogously of all other systems dominated by quantum gravitation). Although this idea remains as the simplest solution of the puzzle not contradicting any natural low, it raised a lot of discussions and controversies, as it necessarily implies fundamental non-unitarity of evolution of the complete system universe + black hole. Alternative solutions include possibility that information is still evaporated through the higher order corrections or that the Hawking evaporation is terminated when the mass of black hole becomes of the order of Planck's mass, leaving bellow the stable remnants encoding complete information on the initial state. However, these alternative solutions of the puzzle bear their deep problems, so the original Hawking's idea is still considered as the most acceptable possibility. It should be pointed out that this (alongside with the collapse of the wave function) is by now the only phenomenon in the physical world where fundamental indeterminism can be manifested. Whether this is really manifested can be only confirmed by the future observable (on detection of the evaporating black holes) and theoretical (on development of the correct theory of the quantum gravitation, exact on the Planck scale) works. In the framework of the idea - originating from Penrose - on the role of the future quantum theory of gravitation in explaining problem of consciousness, the possible causal relation of the indeterminism in consciousness and indeterminism in black holes might be an indirect confirmation of the idea that physical correlates of the mental states could be found only within some theory surpassing the contemporary physical theories.

\section{REFERENCES}

1. A.J. Leggett, Macroscopic quantum systems and the quantum theory of measurement, Prog. Theor. Phys. Suppl. No. 69 (1980), pp. 80-100; A.J. Leggett and A. Garg, Quantum mechanics versus macroscopic realism: Is the flux there when nobody looks?, Phys. Rev. Lett. 54 (1985), pp. 857-860; A.J. Leggett, in Chance and Matter, Les Houches Summer School, 1986, Ch. VI; A.J. Leggett, S. Chakravarty, A.T. Dorsey, M.P.A. Fisher, A. Garg, and W. Zwerger, Dynamics of the dissipative two-state system, Rev. Mod. Phys. 59 (1987), pp. 1-85.

2. Group of authors, Anti-Stress Holistic Handbook: With Fundamentals of Acupuncture, Microwave Resonance Therapy, Relaxation Massage, Aeroionotherapy, Autogenic Training, and Consciousness (IASC, Belgrade, 1999), in Serbian. 
3. N.P. Zalyubovskaya, An Estimation of Effects of Millimeter and Submillimeter Microwawaves upon Various Biological Objects, M.Sc. Thesis in Biological Sciences (Kharkov State University, 1970), in Russian.

4. N.D. Devyatkov, Influence of the millimeter wavelength range electromagnetic radiation upon biological objects, Soviet Physics - Uspekhi 110 (1973), pp. 452-454; see also pp. 455-469 in this volume; N.D. Devyatkov and O. Betskii, eds., Biological Aspects of Low Intensity Millimeter Waves (Seven Plus, Moscow, 1994).

5. Ye.A. Andreyev, M.U. Bely, and S.P. Sit'ko, Manifestation of characteristic eigenfrequencies of human organism, Application for the Discovery to the Committee of Inventions and Discovery at the Council of Ministers of the USSR, No. 32-OT-10609, 22 May 1982, in Russian; S.P. Sit'ko, Ye.A. Andreyev, and I.S. Dobronravova, The whole as a result of self-organization, $J$. Biol. Phys. 16 (1988), pp. 71-73; S.P. Sit'ko and V.V. Gizhko, Towards a quantum physics of the living state, J. Biol. Phys. 18 (1991), pp. 1-10; S.P. Sit'ko and L.N. Mkrtchian, Introduction to Quantum Medicine (Pattern, Kiev, 1994).

6. Z. Jovanović-Ignjatić and D. Raković, A review of current research in microwave resonance therapy: Novel opportunities in medical treatment, Acup. \& Electro-Therap. Res., The Int. J., 24 (1999), pp. 105-125; D. Raković, Z. Jovanović-Ignjatić, D. Radenović, M. Tomašević, E. Jovanov, V. Radivojević, Ž. Martinović, P. Šuković, M. Car, and L. Škarić, An overview of microwave resonance therapy and EEG correlates of microwave resonance relaxation and other consciousness altering techniques, Electro- and Magnetobiology 19 (2000), pp. 193-220 (also presented at 10th Int. Montreux Congress on Stress, Montreux, Switzerland, February/March 1999).

7. H. Frohlich, Long-range coherence and energy storage in biological systems, Int. J. Quant. Chem. 2 (1968), pp. 641-649; H. Frohlich, Theoretical physics and biology, in H. Frohlich, ed., Biological Coherence and Response to External Stimuli (Springer, Berlin, 1991).

8. S.E. Li, V.F. Mashansky, and A.S. Mirkin, Lowfrequency wave and vibrational processes in biosystems, in Vibrational Biomechanics. Using Vibrations in Biology and Medicine, Part I: Theoretical Bases of Vibrational Biomechanics, K.V. Frolov, ed. (Nauka, Moscow, 1989), Ch.3, in Russian; Group of authors, Anti-Stress Holistic Handbook: With Fundamentals of Acupuncture, Microwave Resonance Therapy, Relaxation Massage, Aeroionotherapy, Autogenic Training, and Consciousness (IASC, Belgrade, 1999), in Serbian.

9. G. Keković, D. Raković, M. Satarić, and Dj. Koruga, Model of soliton charge transport through microtubular cytoskeleton in acupuncture system, to be published.

10. E.R. Kandel, S.A. Siegelbaum, and J.H. Schwartz, Synaptic transmission, in E.R. Kandel, J.H. Schwartz, and T.M. Jessell, eds., Principles of Neural Science (Elsevier, New York, 1991), Ch. 9; M.V.L. Benett, L.C. Barrio, T.A. Bargiello, D.C. Spray, E. Hertzberg, and J.C. Ssez, Gap junctions: New tools, new answers, new questions, Neuron 6 (1991), pp. 305-320.

11. A.I. Škokljev, Acupuncturology (ICS, Belgrade, 1976), in Serbian; F.G. Portnov, Electropuncture Reflexotherapeutics (Zinatne, Riga, 1982), in Russian; Y. Omura, Acupuncture Medicine: Its Historical and Clinical Background (Japan Publ. Inc., Tokyo, 1982); C. Xinnong, ed., Chinese Acupuncture and Moxibustion (Foreign Languages Press, Beijing, 1987).

12. J.J. Hopfield, Neural networks and physical systems with emergent collective computational abilities, Proc. Natl. Acad. Sci. USA 79 (1982), pp. 2554-2558.

13. M. Peruš and S.K. Dey, Quantum systems can realize content-addressable associative memory, Appl. Math. Lett. 13 (2000), pp. 31-36; M. Peruš, Neuro-quantum parallelism in mind-brain and computers, Informatica 20 (1996), pp. 173-183.

14. R.P. Feynman and A.R. Hibbs, Quantum Mechanics and Path Integrals (McGraw-Hill, New York, 1965); J.D. Bjorken and S.D. Drell, Relativistic Quantum Theory (McGraw-Hill, New York, 1964).

15. D. Raković, Fundamentals of Biophysics (Grosknjiga, Belgrade, 1994; 1995), Chs. 5-6, in Serbian; D. Raković, Brainwaves, neural networks, and ionic structures: Biophysical model for altered states of consciousness, in D. Raković and Dj. Koruga, eds., Consciousness: Scientific 
Challenge of the 21st Century (ECPD, Belgrade, 1995; 1996); D. Raković, Prospects for conscious brain-like computers: Biophysical arguments, Informatica 21 (1997), pp. 507-516; D. Raković, Transitional states of consciousness as a biophysical basis of transpersonal transcendental phenomena, Int. J. Appl. Sci. \& Computat. 7 (2000), pp. 174-187 (also presented at Int. Conf. Consciousness in Science \& Philosophy, Charleston, IL, USA, November 1998).

16. R. Monroe, Journeys Out of the Body (Doubleday, Garden City, NY, 1971); R.A. Moody, jr., Life after Life (Bantam, New York, 1975); W. Evans Wentz, The Tibetan Book of the Dead (Oxford Univ. Press, London, 1968); V. Nikčević, ed., Life after Life: Experiences of Orthodox Christians (Svetigora, Cetinje, 1995), in Serbian.

17. R.J. Callahan and J. Callahan, Thought Field Therapy and Trauma: Treatment and Theory. Indian Wells, CA: Private Edition, 1996; R.J. Callahan, "The impact of thought field therapy on heart rate variability (HRV)," J. Clin. Psychol., Oct. 2001 (see www.interscience.Wiley.com); Ž. Mihajlović Slavinski, PEAT and Neutralization of Primeval Polarities (Belgrade, 2000), in Serbian.

18. D. Raković and Ž. Mihajlović Slavinski, in preparation (2002).

19. W.R. Adey, Frequency and power windowing in tissue interactions with weak electromagnetic fields, Proc. IEEE 68 (1980), pp. 119-125, and references therein.

20. B.J. Baars, A Cognitive Theory of Consciousness (Cambridge Univ. Press, Cambridge, MA, 1988).

21. K.R. Poper and J.C. Eccles, The Self and Its Brain (Springer, Berlin, 1977).

22. F. Crick, The Astonishing Hypothesis: The Scientific Search for the Soul (Charles Scribner's Sons, New York, 1994).

23. R. Penrose, The Emperor's New Mind (Oxford Univ. Press, New York, 1989); R. Penrose, Shadows of the Mind: A Search for the Missing Science of Consciousness (Oxford Univ. Press, Oxford, England, 1994); R. Penrose, in The Large, the Small and the Human Mind, M. Longair, ed. (Cambridge Univ. Press, Cambridge, England, 1997).

24. C. Tart, ed., Altered States of Consciousness (Academic, New York, 1972).

25. D. Raković and Dj. Koruga, eds., Consciousness: Scientific Challenge of the 21st Century (ECPD, Belgrade, 1995; 1996); Lj. Rakić, D. Raković, Dj. Koruga, and A. Marjanović, eds., Consciousness: Scientific Challenge of the 21st Century, Proc. of ECPD Seminar, 27-28 September 1996, Belgrade, in Serbian; Lj. Rakić, G. Kostopoulos, D. Raković, and Dj. Koruga, eds., Brain \& Consciousness: Proc. ECPD Symposium \& Workshop (ECPD, Belgrade, 1997); V. Jerotić, Dj. Koruga, and D. Raković, eds., Science - Religion - Society (Theological Faculty of Serbian Orthodox Church \& Serbian Ministry of Religions, Belgrade, 2002), in Serbian.

26. A. Shimony, in The Large, the Small and the Human Mind, R. Penrose and A. Shimony, N. Cartwright and S. Hawking, eds. (Cambridge Univ. Press, Cambridge, England, 1995).

27. A. Einstein, B. Podolsky, and N. Rosen, Can quantum-mechanical description of physical reality be considered complete, Phys. Rev. 47 (1935), pp. 777-780.

28. J.S. Bell, Speakable and Unspeakable in Quantum Mechanics (Cambridge University Press, Cambridge, 1987), and references therein; A. Aspect, J. Dalibard, and G. Roger, Experimental test of Bell's inequalities using time-varying analyzers, Phys. Rev. Lett. 49 (1982), pp. 18041807; W. Tittel, J. Brendel, H. Zbinden, and N. Gisin, Violation of Bell inequalities by photons more than 10km apart, Phys. Rev. Lett. 81 (1998), pp. 3563-3566.

29. M. Tegmark, Importance of quantum decoherence in brain processes, Phys. Rev. E 61 (2000), pp. 4194-4206.

30. J. von Neumann, Mathematical Foundations of Quantum Mechanics (Princeton Univ. Press, Princeton, NJ, 1955).

31. E. Wigner, Remarks on the mind-body problem, in Symmetries and Reflections (Indiana Univ. Press, Bloomington, 1967).

32. H.P. Stapp, Quantum theory and the role of mind in nature, Found. Phys. 31 (2001), pp. 14651499; H. Stapp, Mind, Matter, and Quantum Mechanics (Springer, New York \& Berlin, 1993).

33. N. Bohr, Can quantum-mechanical description of physical reality be considered complete, Phys. 
Rev. 48 (1935), pp. 696-702; P.A.M. Dirac, at Solvay Conf. Electrons et Photons: Rapports et Discussions du Cinquieme Conseil de Physique (Gauthier-Villars, Paris, 1928); W. Heisenberg, The representation of nature in contemporary physics, Daedalus 87 (1958), pp. 95-108.

34. M.S. Morris, K.S. Thorne, and U. Yurtsever, Wormholes, time machines, and the weak energy condition, Phys. Rev. Lett. 61 (1988), pp. 1446-1449; M. Visser, Quantum wormholes, Phys. Rev. D 43 (1991), pp. 402-409; K.S. Thorne, Black Holes and Time Warps: Einstein's Outrageous Legacy (Picador, London, 1994), and references therein.

35. R.J. Jahn and B.J. Dunne, Margins of Reality (Harcourt Brace, New York, 1988), and many archival publications and technical reports by PEAR (Princeton Engineering Anomalies Research); see www.princeton.edu/ rdnelson/pear.html.

36. W.A. Tiller, W.E. Dibble, Jr., and M.J. Kohane, Exploring robust interactions between human intention and inanimate/animate systems, Ditron Preprint (presented at Int. Conf. Toward a Science of Consciousness - Fundamental Approaches, UN Univ., Tokyo, Japan, May 1999), and references therein.

37. V.P. Kaznacheev and A.V. Trofimov, Cosmic Consciousness of Humanity (Elendis-Progress, Tomsk, 1992), and references therein.

38. www.dukenews.duke.edu 1998, 9 Nov., News (Medical Center News Office, Duke University, NC, USA) - Results of this pilot study on 150 patients were intriguing enough to initiate wider investigations in several american medical centers; Overview of the previous similar studies can be found in the book of L. Dossey, Healing Words: The Power of Prayer and The Practice of Medicine (Harper Paperbacks, New York, 1993).

39. M. Dugić, D. Raković, and M.M. Ćirković, On a physical metatheory of consciousness, in I. Kononenko, ed., New Science of Consciousness: Proc. IS'2000 (Information Society, Ljubljana, 2000); M. Dugić, M.M. Ćirković, and D. Raković, On a possible physical metatheory of consciousness, Open Systems \& Information Dynamics 9 (2002), pp. 153-166.

40. W.H. Zurek, Decoherence and the transition from quantum to classical, Physics Today 48 (1991), pp. 36-46.

41. R. Omnes, The Interpretation of Quantum Mechanics (Princeton Univ. Press, Princeton, 1994).

42. D. Giulini, E. Joos, C. Kiefer, J. Kupsch, I.-O. Stamatescu, and H.D. Zeh, Decoherence and the Appearance of a Classical World in Quantum Theory (Springer, Berlin, 1996).

43. D. Raković and M. Dugić, A critical note on the role of the quantum mechanical "collapse" in quantum modeling of consciousness, Informatica 26 (2002), pp. 85-90.

44. M. Dugić, On the occurrence of decoherence in nonrelativistic quantum mechanics, Physica Scripta 53 (1996), pp. 9-17; M. Dugić, On diagonalization of the composite-system observable separability, Physica Scripta 56 (1997), pp. 560-565; M. Dugić, Contribution to the Foundation of the Theory of Decoherence in Nonrelativistic Quantum Mechanics, Ph.D. Thesis (Faculty of Science, University of Kragujevac, 1997), in Serbian.

45. K. Wilber, The Atman Project (Quest, Wheaton, IL, 1980); P. Vujičin, in Consciousness: Scientific Challenge of the 21st Century, D. Raković and Dj. Koruga, eds. (ECPD, Belgrade, 1995; 1996), and references therein.

46. J.S. Hagelin, Is consciousness the unified field? A field theorist's perspective, Modern Sci. \& Vedic Sci. 1 (1987), pp. 29-88, and references therein.

47. J.D. Barrow and F.J. Tipler, The Anthropic Cosmological Principle (Oxford Univ. Press, New York, 1986).

48. H. Everett III, "Relative state" formulation of quantum mechanics, Rev. Mod. Phys. 29 (1957), pp. 454-462.

49. B. Carter, in Physical Cosmology and Philosophy, J. Leslie, ed. (Macmillan, London, 1990).

50. D. Amit, Modeling Brain Functions: The World of Attractor Neural Nets (Cambridge Univ. Press, Cambridge, MA, 1989); M. Peruš and P. Ečimovič, Memory and pattern recognition in associative neural networks, Int. J. Appl. Sci. \& Computat. 4 (1998), pp. 283-310.

51. H. Haken, Synergetic Computers and Cognition: A Top-Down Approach to Neural Nets (Springer, Berlin, 1991). 
52. J.G. Sutherland, Holographic model of memory, learning and expression, Int. J. Neural. Sys. 1 (1990), pp. 256-267.

53. A. Kitaev, A. Shen', and M. Vyalii, Classical and Quantum Computations (MCNMO, Moscow, 1999), in Russian; C. Bennett, G. Brassard, C. Crepeau, R. Jozsa, A. Peres, W. Wootters, Teleporting an unknown quantum state via dual classical and Einstein-Podolsky-Rosen channel, Phys. Rev. Lett. 70 (1993), pp. 1895-1899.

54. D. Raković, On brain's neural networks and brainwaves modeling: Contextual learning and psychotherapeutic implications, in B. Lithgow and I. Cosic, eds., Biomedical Research in 2001: 2nd Proc. IEEE/EMBS (Vic) (IEEE/EMBS Victorian Chapter, Melbourne, Australia, 2001).

55. Y.J. Ng, From computation to black holes and space-time foam, Phys. Rev. Lett. 86 (2001), pp. 2946-2949.

56. D. Witztum, E. Rips, and Y.Rosenberg, Equidistant letter sequences in The Book of Genesis, Statistical Science 9 (1994), pp. 429-438; a popular survey of mathematical discovery of this extraordinary code is given in the book: M. Drosnin, The Bible Code (Simon \& Schuster, New York, 1997). The fascinating possibility that history of Civilization is preferently coded in The Old Testament revealed to Moses on the Mount Sinai 3000 years ago, might be interpreted as a consequence of the quantum-holographic nature of the cosmic collective consciousness (God!?) and its every original manifestation (including The Old Testament, as well as every individual consciouness) - which still does not mean the strict determinism of the History by the existing state of collective consciousness, whose memory attractors could be reprogramed by merciful prayer for the others thus removing interpersonal loads of the quantum-holographic neural network of collective consciousness - so leaving the room for free will and influence on future preferences $[15,59]$.

57. R.G. Jahn, The persistent paradox of psychic phenomena: An engineering perspective, Proc. IEEE 70 (1982), pp. 136-170, and references therein.

58. J. Vlahos, Orthodox Psychotherapy: The Holy Fathers Science (Orthodox Missionary School of St. Alexander Nevskiy Church, Belgrade, 1998), Serbian translation from Greek.

59. D. Raković, Biophysical frontiers of holistic psychosomatics, in I. Kononenko, ed., New Science of Consciousness: Proc. IS'2000 (Information Society, Ljubljana, Slovenia, 2000); D. Raković, Consciousness-based biophysical frontiers of complementary medicine, in B. Lithgow and I. Cosic, eds., Biomedical Research in 2001: 2nd Proc. IEEE/EMBS (Vic) (IEEE/EMBS Victorian Chapter, Melbourne, Australia, 2001); D. Raković, Hopfield-like quantum associative neural networks and (quantum)holistic psychosomatic implications, in B. Reljin, ed., Proc. NEUREL2002 (IEEE Yugoslavia Section, Belgrade, 2002).

60. J.H. Schultz, Das Autogene Training (Thieme, Stuttgart, 1951), 7th ed.; H. Lindemann, Autogenic Training (Prosvjeta, Zagreb, 1976), Serbocroat translation from German; Lj. Mirković, Autogenic Training - New Generation (NNK, Belgrade, 1998), in Serbian; A. Poro, Encyclopedia of Psychiatry (Nolit, Belgrade, 1990), Serbian translation from French.

61. S. Milenković, Values of Contemporary Psychotherapy (Prometej, Novi Sad, 1997), in Serbian.

62. V. Jerotić, Individuation and (or) Deification (Ars Libri, Belgrade \& Public University Library, Priština, 1998), in Serbian; cf. especially Ch.7: Confession in Christian Churches and Psychotherapy.

63. J.D. Bekenstein, Black holes and entropy, Phys. Rev. D 7 (1973), pp. 2333-2346.

64. S.W. Hawking, Particle creation by black holes, Commun. Math. Phys. 43 (1975), pp. 199-220.

65. C.B. Collins and S.W. Hawking, Why is the universe isotropic, Astrophys. J. 180 (1973), pp. 317-334.

66. S.W. Hawking, The unpredictability of quantum gravity, Commun. Math. Phys. 87 (1982), pp. 395-415.

67. S.B. Giddings, Black holes and massive remnants, Phys. Rev. D 46 (1982), pp. 1347-1352. 